\title{
Prevalencia y características del abuso sexual infantil en estudiantes universitarios españoles ${ }^{\text {is }}$
}

\author{
Noemí Pereda*, Maria Forns \\ Departament de Personalitat, Avaluació i Tractament Psicològics, Facultat de Psicologia, \\ Universitat de Barcelona, Passeig Vall d'Hebron, 171, 08035 Barcelona, Spain
}

Received 21 April 2006; accepted 24 August 2006

Available online 6 April 2007

\section{Resumen}

Objetivo: Este estudio pretende actualizar y profundizar en la prevalencia y características del abuso sexual infantil en estudiantes universitarios españoles.

Método: Se ha estudiado una muestra $(N=1.033)$ de estudiantes matriculados en la Universidad de Barcelona durante el curso 2001/2002. Los datos fueron obtenidos mediante el cuestionario Traumatic Life Events Questionnaire (TLEQ; Kubany y Haynes, 2001). En este cuestionario el abuso sexual se define como el contacto sexual no deseado (a) entre un menor de 13 años y alguien al menos cinco años mayor, (b) entre un menor de 13 años y alguien de similar edad mediante coerción, y (c) entre un menor de 18 años y otro individuo contra su voluntad.

Resultados: La prevalencia del abuso sexual antes de los 18 años se sitúa en un 17,9\% (un 14,9\% antes de los 13 años y un $3 \%$ entre los 13 y los 18 años). Un 15,5\% de los varones y un 19\% de las mujeres manifiestan haber sufrido esta experiencia. Se observan diferencias en el tipo de agresor y las características del abuso en función de la edad de inicio de éste y del sexo de las víctimas. Destaca el elevado porcentaje de penetración, tanto en varones como en mujeres antes de los 13 años $(26,7 \%$ y 42,1\%) y después de esta edad (27,3\% y $25 \%)$.

Discusión: La actualización de las cifras de prevalencia del abuso sexual infantil permite confirmar que éste sigue siendo un problema extendido en la sociedad española, siendo necesarios programas de prevención y ayuda para el elevado número de víctimas.

(C) 2007 Elsevier Ltd. All rights reserved.

Keywords: Prevalencia; Abuso sexual infantil; Estudiantes universitarios; España

\footnotetext{
This study was supported in part by the grant SEJ 2005-09144-C02-01 from the Spanish Department of Education \& Science.

${ }^{*}$ Corresponding author.
} 
El abuso sexual infantil ha sido considerado uno de los problemas de salud pública más graves que tiene que afrontar la sociedad y, especialmente, los niños y jóvenes (MacMillan, 1998). Los estudios realizados al respecto confirman que se trata de un problema mucho más extendido de lo previamente estimado y que incluso las tasas de prevalencia más bajas incluyen a un gran número de víctimas que debe tenerse en cuenta.

El descubrimiento del abuso sexual infantil como maltrato frecuente y con importantes y perdurables efectos psicológicos, tanto a corto como a largo plazo, ha dado lugar en la última década a un notable crecimiento de los estudios sobre este tema tanto a nivel nacional como internacional. Destacan los estudios realizados con muestras jóvenes, principalmente con estudiantes universitarios. Estos estudios se plantean como solución, al menos parcial, a los problemas de recuerdo y a la presencia de distorsiones que suelen producirse cuando se trabaja, en estudios retrospectivos, con adultos mayores (Halperin et al., 1996). La utilización de muestras formadas por estudiantes universitarios también se ha criticado. Principalmente, se ha argumentado que los universitarios se encuentran funcionando a un nivel cognitivo superior a la media y que, probablemente, entre ellos no se incluyan demasiados casos de abuso sexual infantil grave (Dhaliwal, Gauzas, Antonowicz y Ross, 1996). No obstante, los estudios realizados con este tipo de muestras demuestran que las cifras no son de ningún modo bajas y que, en muchos casos, contienen abusos sexuales severos como la penetración (Bendixen, Muus y Schei, 1994; Oaksford y Frude, 2001; Rind, Tromovitch y Bauserman, 1998).

Si bien en países como Estados Unidos el estudio de este tema se inició hace ya algunos años (Kempe, 1978), en España, el aislamiento sociopolítico y el escaso desarrollo de los sistemas de protección social durante el período de la dictadura, han producido un retraso en el estudio de este tema y, sobre todo, en el conocimiento y la sensibilización social al respecto (Arruabarrena y De Paúl, 1999).

Durante la última década han surgido importantes estudios que han favorecido el avance del conocimiento sobre este problema, así como el establecimiento de datos nacionales que han permitido la comparación con el resto de países occidentales. Este creciente interés tanto a nivel profesional como en los medios de comunicación, ha provocado un mayor conocimiento del problema y una mayor sensibilidad social al respecto (Echeburúa y Guerricaechevarría, 2000). A pesar de ello, los estudios epidemiológicos realizados en España siguen siendo escasos. Los datos de prevalencia obtenidos en nuestro país pertenecen al único estudio nacional realizado hasta el momento por López (1994), recogido por Finkelhor (1994) en su revisión. Mediante muestreo aleatorio estratificado proporcional por comunidades autónomas, edad y sexo, los autores obtuvieron que un 18,9\% de las 1.821 personas entrevistadas de entre 18 y 60 años manifestaron haber sufrido abuso sexual en algún momento de su infancia. En los varones este porcentaje fue del 15,2\% y en las mujeres del 22,5\%. Otro de los escasos estudios de prevalencia del maltrato infantil realizados en España es el llevado a cabo por De Paúl, Milner y Múgica (1995). Estos autores realizaron una réplica del estudio de Milner, Robertson y Rogers (1990) llevado a cabo con universitarios norteamericanos, comparando la prevalencia de maltrato infantil y, dentro de éste, de abuso sexual con y sin contacto físico. Los autores observaron que, de una muestra de 426 estudiantes de psicología de la Universidad del País Vasco con una media de edad de 20,6 años ( $D T=3,2)$, un 13,4\% (9,7\% de los varones y 14,9\% de las mujeres) manifestaban haber sufrido abuso sexual infantil (un 3,9\% de los varones y un 6,4\% de las mujeres antes de los 13 años; un 2,9\% de los varones y un 3,7\% de las mujeres después de los 13 años; y un 2,9\% de los varones y un 4,7\% de las mujeres antes y después de los 13 años).

Estos dos trabajos presentan diferencias metodológicas que pueden haber influido en los datos obtenidos. El estudio de López (1994) presenta un elevado porcentaje de abuso sexual en población general, abarca un amplio muestreo con representación de las diversas comunidades autónomas y una 
gran amplitud en el rango de edad de los sujetos analizados. El trabajo de De Paúl et al. (1995), por su parte, ofrece unos porcentajes de abuso sexual inferiores, una muestra más limitada y una población objeto de estudio más homogénea. Desde la elaboración de estos dos estudios ha transcurrido más de una década. La situación sociocultural española durante este último decenio ha mejorado notablemente, con la consolidación de la democracia y la decidida incorporación a la Unión Europea. Así, se ha considerado de utilidad proceder a una actualización de estos datos, que puedan clarificar las diferencias detectadas.

Este trabajo tiene, pues, como objetivo actualizar los datos de prevalencia y de las características del abuso sexual infantil en una muestra de estudiantes universitarios de la Universidad de Barcelona, siendo el primer trabajo de prevalencia llevado a cabo con muestra universitaria en la comunidad autónoma de Catalunya.

\section{Método}

\section{Sujetos}

La muestra se compone de 1.033 estudiantes universitarios de entre 18,0 y 30,6 años $(M=21,71$; $D T=2,63)$, pertenecientes a distintas facultades y centros adscritos de la Universidad de Barcelona con 317 varones $(30,7 \%)$, cuya media de edad es de 21,9 años $(D T=2,67)$; y 716 mujeres $(69,3 \%)$, cuya media de edad es de 21,6 años $(D T=2,61)$. La selección de la muestra siguió criterios de proporcionalidad en función del número de estudiantes matriculados en las distintas Divisiones Académicas y centros adscritos de la Universidad de Barcelona en el curso académico 2001/2002, situándose la proporcionalidad de la muestra en un valor promedio de 1,75\%. La selección de los centros fue aleatoria de entre las distintas facultades pertenecientes a cada División Académica y centros adscritos de la Universidad de Barcelona en el curso académico 2001/2002.

\section{Materiales}

La identificación y la caracterización del abuso sexual infantil se realizaron a partir de la selección de los tres ítems referidos a este estresor del cuestionario Traumatic Life Events Questionnaire (TLEQ) (Kubany y Haynes, 2001), que estaba siendo empleado en un estudio sobre la vivencia de acontecimientos estresantes en estudiantes universitarios.

El cuestionario evalúa la vivencia de 22 acontecimientos vitales potencialmente traumáticos, siguiendo la definición del DSM para acontecimientos traumáticos (American Psychiatric Association, 2002). Los acontecimientos del cuestionario se presentan en orden gradual desde aquellos estresores externos al individuo (por ejemplo, desastres naturales, accidentes de tráfico) a aquellos más personales (por ejemplo, ser testigo de violencia doméstica o ser víctima de maltrato físico infantil), lo que permite reducir el posible rechazo de los encuestados a aquellas preguntas más intrusivas.

En cuanto al abuso sexual, el cuestionario permite diferenciar entre abuso sexual infantil antes de los 13 años por alguien al menos cinco años mayor (abuso tipo I) y antes de los 13 años por alguien de similar edad mediante el uso de la fuerza, las amenazas, el engaño, la inconsciencia o el abuso de autoridad (abuso tipo II), correspondiéndose con las definiciones presentadas en gran parte de la literatura sobre el tema (Finkelhor y Hotaling, 1984; López, 1994). Los ítems también identifican el Abuso Sexual Adolescente, 
definido como el contacto sexual entre un adolescente de 13 a 18 años y otro individuo, contra sus deseos o sin su consentimiento.

Por otra parte, la diferenciación establecida entre abuso sexual infantil con inicio anterior y posterior a los 13 años es útil para nuestro estudio ya que permite equipararlos a la edad mínima establecida en el código penal español para aceptar el consentimiento de una relación sexual (Título VIII Cap. II. De los abusos sexuales, art. 181/2).

Los ítems también permiten obtener información sobre el agresor, el uso de fuerza o amenazas, la presencia de heridas graves en la víctima como resultado del abuso y la vivencia de penetración oral, anal o vaginal.

Los estudios de fiabilidad test-retest del instrumento, llevados a cabo por Kubany y Haynes (2001) para las experiencias de abuso sexual antes de los 13 años por alguien al menos cinco años mayor ofrecen unos índices $\kappa$ de,70 a,90. Los autores reportan también una adecuada validez de contenido, convergente y discriminante.

\section{Diseño}

El diseño que sigue este trabajo comparte las características de los trabajos de investigación retrospectivos de naturaleza correlacional y de los trabajos cuasiexperimentales.

\section{Procedimiento}

Selección de la muestra. Una vez seleccionadas aleatoriamente las facultades de cada División Académica y los centros adscritos de la Universidad de Barcelona en el curso académico 2001/2002, se estableció contacto con los respectivos Jefes de Estudio. Si se denegaba la petición de colaboración $(3,85 \%)$ o no se obtenía respuesta del Jefe de Estudio $(38,5 \%)$, se seleccionaba, de forma aleatoria, otra facultad perteneciente a la misma División. Las aulas, dentro de cada facultad y centro, se seleccionaban aleatoriamente en función de las posibilidades del centro, según los criterios de proporcionalidad establecidos.

Adaptación del TLEQ. El cuestionario fue traducido por psicólogos expertos en la lengua inglesa y se realizó una backtranslation por otro psicólogo nativo del Reino Unido (porcentaje de acuerdo entre los ítems: 96\%). La correspondencia entre los cuestionarios originales y la traducción española realizada fue evaluada por un experto en psicología clínica, que confirmó la adecuación del contenido de los ítems de las versiones españolas. Se enviaron copias del cuestionario traducido a sus autores originales quienes dieron su conformidad con la adaptación y la estructura del cuestionario (Kubany y Haynes, 2001).

Aplicación de los instrumentos. Se explicó el objetivo del estudio a los jefes de estudio de las facultades y centros adscritos seleccionados previamente, obteniéndose su consentimiento verbal. Cada facultad proporcionó una o dos aulas con un número de estudiantes indeterminado, también seleccionadas de forma aleatoria. El día de la aplicación se realizó una breve presentación a los estudiantes en la que se les pedía su colaboración para responder a una serie de cuestiones, destacando su confidencialidad y la sinceridad requerida. Se explicó a los estudiantes que el objetivo del estudio era evaluar la vivencia de acontecimientos traumáticos en población universitaria. No se hizo referencia explícita a la experiencia de abuso sexual infantil. Todos los estudiantes colaboraron y ninguno de ellos interrumpió la aplicación 
abandonando la sala. Tres investigadoras licenciadas en psicología acudían a las sesiones, facilitando la aplicación de los cuestionarios y atendiendo a todas las posibles dudas planteadas por los estudiantes. Tras la respuesta de los estudiantes a los cuestionarios se les ofrecía un teléfono de atención, así como la posibilidad de una atención individual y, en caso necesario, diversas sesiones de orientación con una de las psicólogas investigadoras del estudio. Únicamente dos estudiantes requirieron de este servicio. Tras finalizar el estudio se entregó un resumen global con los resultados más importantes de cada facultad y centro adscrito a sus respectivos jefes de estudio.

\section{Resultados}

Se presenta a continuación la prevalencia del abuso sexual infantil en estudiantes universitarios diferenciando entre abuso sexual con inicio anterior a los 13 años, independientemente de la edad del agresor y abuso sexual con inicio posterior a los 13 años pero anterior a los 18 años.

Dentro de la primera categoría se incluyen: (a) aquellos casos que manifestaron haber sufrido abuso sexual antes de los 13 años por un agresor al menos cinco años mayor (tipo I) y (b) aquellos casos que manifestaron haber sufrido abuso sexual antes de los 13 años por un agresor de similar edad (tipo II). Aquellos casos que manifestaron haber sufrido abuso sexual antes de los 13 años por ambos tipos de agresores sólo fueron contabilizados una vez.

La segunda categoría incluye aquellos casos que manifestaron haber sufrido contacto sexual no deseado después de los 13 pero antes de los 18 años (excluyéndose aquellos casos cuya respuesta a este ítem fuese una prolongación del abuso iniciado antes de los 13 años por un mismo agresor, que fue contabilizado en la primera categoría).

En la Tabla 1 se observan los porcentajes correspondientes a estas dos categorías según el sexo de los estudiantes.

La experiencia de abuso sexual antes de los 13 años es frecuente tanto en estudiantes varones (12,0\%) como, especialmente, en estudiantes mujeres $(16,2 \%)(z=1,75 ; p=, 040 ; O R=1,42)$. El porcentaje de abuso sexual entre los 13 y los 18 años, alcanza el 3,5\% en varones y el 2,8\% en mujeres, no existiendo diferencias entre ambos $\operatorname{sexos}(z=, 59 ; p=, 278)$ si bien los varones presentan un mayor riesgo de experimentar este tipo de abuso $(O R=1,25)$.

En total, los resultados indican que un $17,9 \%$ del total de la muestra universitaria estudiada (15,5\% de los varones y $19 \%$ de las mujeres) manifiestan haber sufrido abuso sexual con contacto físico antes de los 18 años. De ellos, un 14,9\% ha sido víctima del abuso antes de los 13 años y un 3\% después de los 13 pero antes de los 18 años.

Para profundizar con más detalle en estos resultados, se desglosaron las categorías anteriores en las siguientes: (a) haber sufrido abuso sexual antes de los 13 años por alguien al menos cinco años mayor

Table 1

Prevalencia del abuso sexual antes y después de los 13 años

\begin{tabular}{lccc}
\hline & Abuso sexual antes de los 13 años $(\%)$ & Abuso sexual entre los 13 y los 18 años $(\%)$ & Total $(\%)$ \\
\hline Varones & 12,0 & 3,5 & 15,5 \\
Mujeres & 16,2 & 2,8 & 19,0 \\
Total & 14,9 & 3,0 & \\
\hline
\end{tabular}


Table 2

Prevalencia del abuso sexual infantil desglosada

\begin{tabular}{lcccc}
\hline & $\begin{array}{c}\text { Abuso sexual antes } \\
\text { de los 13 años I (\%) }\end{array}$ & $\begin{array}{c}\text { Abuso sexual antes de } \\
\text { los 13 años II (\%) }\end{array}$ & $\begin{array}{c}\text { Abuso sexual entre los } \\
13 \text { y los } 18 \text { años }(\%)\end{array}$ & $\begin{array}{c}\text { Combinaciones de } \\
\text { abuso sexual }(\%)\end{array}$ \\
\hline Varones & 6,0 & 4,1 & 3,5 & 1,9 \\
Tujeres & 8,7 & 2,9 & 2,8 & 4,6 \\
Total & 7,8 & 3,3 & 3,0 & 3,8 \\
\hline
\end{tabular}

(tipo I), (b) haber sufrido abuso sexual antes de los 13 años por alguien de similar edad (tipo II), (c) haber sufrido abuso sexual después de los 13 años pero antes de los 18, y (d) haber sufrido formas mixtas de abuso (abuso sexual antes de los 13 años por un agresor al menos cinco años mayor y simultáneamente por un agresor de similar edad o abuso sexual antes de los 13 años y también después de los 13 pero antes de los 18 años).

La Tabla 2 pone de relieve que un 7,8\% del total de la muestra manifestó haber sufrido abuso sexual antes de los 13 años por un agresor al menos cinco años mayor. Esta es la categoría de abuso sexual más frecuente, no existiendo diferencias significativas entre varones y mujeres $(z=1,47 ; p=, 071)$, si bien las mujeres muestran un mayor riesgo de sufrir esta tipología de abuso sexual $(O R=1,49)$.

La vivencia de abuso sexual antes de los 13 años por alguien de similar edad y después de los 13 pero antes de los 18 años presenta porcentajes similares, oscilando entre un 4,1\% y un 2,9\% del total de la muestra, respectivamente. No existen diferencias significativas entre varones y mujeres víctimas de abuso sexual antes de los 13 años por un agresor de similar edad $(z=, 97 ; p=, 166)$, si bien los varones presentan un mayor riesgo de experimentar este tipo de abuso sexual $(O R=1,41)$. Tampoco existen diferencias entre sexos en el abuso sexual experimentado después de los 13 años pero antes de los 18 años $(z=, 59$; $p=, 278)$, si bien los varones muestran un mayor riesgo ante este tipo de abuso sexual $(O R=1,25)$.

Destaca que en un 3,8\% del total de la muestra, los estudiantes manifiestan haber sufrido más de una experiencia de abuso sexual por agresores distintos, observándose diferencias entre sexos $(z=2,11$; $p=, 017)$, con un mayor riesgo de experimentar distintos tipos de abuso sexual en estudiantes mujeres $(\mathrm{OR}=2,50)$.

\section{Abuso sexual antes de los 13 años: agresor y características}

En el estudio, se ha contemplado la figura del agresor bajo las siguientes categorías: (a) desconocido, (b) padre, madre o cuidador, (c) amigo o conocido y (d) otro familiar. Los resultados expresados en la Tabla 3 indican que el agresor es predominantemente un amigo o conocido de la víctima en víctimas de sexo masculino $(z=2,80 ; p=, 002)$ y un familiar en víctimas de sexo femenino $(z=2,40 ; p=, 008)$.

Table 3

Tipología del agresor antes de los 13 años, según el sexo de los estudiantes

\begin{tabular}{lcccc}
\hline & \multicolumn{2}{l}{ Agresor } & & \\
\cline { 2 - 5 } & Desconocido (\%) & Padre, madre o cuidador $(\%)$ & Amigo o conocido (\%) & Familiar $(\%)$ \\
\hline Varones & 23,7 & 2,6 & 65,8 & 23,7 \\
Mujeres & 29,3 & 6,7 & 39,6 & 45,7 \\
\hline
\end{tabular}


Table 4

Tipología del agresor después de los 13 años, según el sexo de los estudiantes

\begin{tabular}{lccccc}
\hline & \multicolumn{1}{l}{ Agresor } & & & & \\
\cline { 2 - 6 } & Desconocido (\%) & Padre, madre o cuidador (\%) & Familiar (\%) & Amigo o conocido (\%) & Pareja (\%) \\
\hline Varones & 54,5 & 0 & 9,1 & 27,3 & 9,1 \\
Mujeres & 20 & 0 & 15 & 35 & 35 \\
\hline
\end{tabular}

Asimismo, cabe destacar que en víctimas de sexo femenino el porcentaje de agresores amigos o conocidos $(39,6 \%)$ y el de familiares $(45,7 \%)$ es muy similar. No obstante, los individuos víctimas de abuso sexual pueden haber sufrido esta experiencia por parte de agresores distintos, de modo que las categorías de agresores no son mutuamente excluyentes y la suma de porcentajes puede ser superior a 100.

Las características de este tipo de abuso sexual con inicio anterior a los 13 años en cuanto a uso de fuerza y/o amenazas por parte del agresor, presencia de heridas graves en la víctima como resultado del abuso y penetración (oral, anal o vaginal), se detallan a continuación.

Los agresores suelen usar un tipo de violencia que no deja heridas graves como resultado del abuso sexual, ni en víctimas de sexo masculino (0\%) ni femenino (0\%). Sin embargo se detecta el uso de fuerza y amenazas en el 23,7\% de varones y en el 18,1\% de mujeres. La penetración (oral, anal o vaginal) es elevada tanto en mujeres $(26,7 \%)$, como especialmente en varones $(42,1 \%)$, presentándose en un porcentaje significativamente alto en el caso de víctimas de sexo masculino $(z=1,79 ; p=, 037)$.

\section{Abuso sexual después de los 13 pero antes de los 18 años: agresor y características}

Como muestra la Tabla 4, existe un cambio muy notorio en la tipología del agresor en víctimas de abuso sexual con inicio posterior a los 13 años. Los porcentajes de agresores amigos o conocidos y familiares descienden, aunque continúan siendo relevantes, y se incrementa el número de agresores desconocidos, cuyo porcentaje es especialmente elevado en víctimas de sexo masculino $(z=1,97 ; p=, 024)$. Aparecen también como agresores frecuentes las propias parejas de las víctimas, especialmente en estudiantes de sexo femenino. Recordar que la suma de porcentajes puede ser superior a 100 debido a que un mismo sujeto puede haber sufrido abuso sexual por dos o más agresores distintos.

También se detecta un cambio substancial respecto a las características del abuso sexual. En víctimas de abuso sexual con inicio posterior a los 13 años se incrementa el uso de fuerza o amenazas sobre las estudiantes de sexo femenino $(30 \% ; z=2,02 ; p=, 021)$, si bien ninguna de ellas dice haber sufrido heridas graves como consecuencia del abuso sexual (0\%). Los varones manifiestan no haber sufrido la fuerza y las amenazas del agresor $(0 \%)$, ni tampoco heridas graves como resultado $(0 \%)$. Los porcentajes de penetración en varones se reducen en esta categoría $(27,3 \%)$, si bien siguen siendo muy elevados. En las mujeres, el porcentaje de penetración no varía significativamente en función de la edad de inicio del abuso $(25 \%)$.

\section{Discusión}

La prevalencia de abuso sexual infantil en estudiantes universitarios españoles se corresponde con los resultados obtenidos en estudios realizados con muestras universitarias pertenecientes a países europeos, 
siendo ligeramente superiores a éstos en el caso de las mujeres y especialmente significativos en el caso de los varones (Bendixen et al., 1994; Oaksford y Frude, 2001). Las cifras de prevalencia obtenidas en este trabajo, en comparación con las de estudios de prevalencia publicados en España, hace más de diez años, muestran un aumento de los porcentajes del $4 \%$ en el caso de las mujeres y de más del $5 \%$ en los varones estudiantes universitarios (De Paúl et al., 1995); y porcentajes muy similares a los obtenidos con muestras pertenecientes a la población general (López, 1994). Este incremento es más relevante si se toma en cuenta que en el presente estudio sólo se han considerado como conductas de abuso sexual aquellas en las que ha habido contacto físico.

Como posibles explicaciones del incremento de la prevalencia del abuso sexual cabe destacar aspectos psicológicos, relacionados con las víctimas, y aspectos metodológicos, referidos a diferencias entre los estudios.

Entre los primeros, Runyan (1998) sugiere que la facilidad o dificultad con que los participantes a un estudio explican sus experiencias sexuales y, especialmente aquellas referidas a abuso sexual, varía dramáticamente entre culturas afectando a los estudios de prevalencia. En nuestro caso, el nivel educativo universitario de la muestra analizada les podría haber proporcionado una mayor concienciación acerca de su experiencia y de la necesidad de no negar, y poder relatar, los conflictos acaecidos. Otra posible explicación podría ser que los estudiantes españoles tuvieran una mayor facilidad (expresividad, desinhibición o realismo) para relatar sus experiencias, fuesen cuales fuesen. Las diferencias encontradas con el trabajo de De Paúl et al. (1995), también con muestra universitaria española, podrían relacionarse con diferencias socioculturales (i.e., mayor sensibilización social hacia el maltrato infantil, mayor libertad en temas referidos a la sexualidad) en las cohortes objeto de estudio.

Por otro lado, las diferencias encontradas también podrían estar relacionadas con diferencias metodológicas como sostiene Finkelhor (1994), más que con diferencias reales entre distintas poblaciones. Entre las diferencias metodológicas de mayor relevancia cabe citar la delimitación del término de abuso, en sí mismo, y de las características que lo definen. En el trabajo presentado el acontecimiento del abuso sexual en la infancia ha sido evaluado a la par que otros múltiples acontecimientos estresantes, siendo anónima la respuesta al cuestionario. Estas dos condiciones de aplicación pueden haber facilitado la respuesta de los estudiantes relativa a la revelación del abuso sexual, y reducido el rechazo que las cuestiones excesivamente focalizadas sobre este tema suele provocar en los participantes de los estudios (Briere, 1992), especialmente en el caso de los varones (Romano y De Luca, 2001; Violato y Genuis, 1993).

Así, el aumento en las cifras de prevalencia obtenidas en este trabajo, en relación a otros estudios realizados con muestras similares, puede deberse tanto a diferencias metodológicas (muestras de distinto origen, instrumentos distintos, cohortes diferentes, distinta delimitación del concepto y de las características del abuso, forma de recogida de datos), como a una mayor sensibilización social respecto a este problema que facilitaría que la víctima decida revelar experiencias de este tipo; aunque no cabe descartar la posibilidad de una mayor ocurrencia real del abuso sexual infantil. Las características de este trabajo no permiten establecer una única explicación.

De los estudios españoles de prevalencia del abuso sexual se deduce que, para los varones, el porcentaje de abuso es significativamente más elevado al encontrado en el resto de países occidentales con muestras similares. López (1994) justifica estos datos debido, en parte, al alto porcentaje de víctimas varones que sufrieron abuso sexual infantil por parte de educadores y religiosos durante el período situado entre 1939 y 1977. Sin embargo, en la muestra analizada en el presente estudio, ésta no parece ser la única explicación, puesto que las edades de los estudiantes no se corresponden con ese período histórico y 
la situación psicosocial ha cambiado mucho en España desde el advenimiento de la democracia (menor escolarización en internados, incremento de profesionales educativos seglares, mayor libertad ideológica). A pesar de todos estos cambios, los resultados obtenidos en este trabajo indican un ligero aumento en la prevalencia del abuso sexual infantil, tanto en mujeres (aproximadamente un 3\%), como en varones (un 2\%), si se considera únicamente el porcentaje de abuso sexual con contacto físico encontrado por López (1994). Ello invita a reflexionar sobre la procedencia del perpetrador. Los datos obtenidos en el presente trabajo sugieren que éste se halla en ámbitos diversos, tanto intra como extrafamiliares. Desde la perspectiva educativa, la reciente experiencia eclesiástica americana en relación a la revelación de abusos sexuales obliga a no desestimar el riesgo indicado por López (1994) pero, además, cabe contemplar un origen vinculado a ámbitos educativos seglares, familiares y de los propios pares.

Una mención especial requiere la prevalencia de abuso sexual cometido por un agresor de similar edad a la víctima. López (1994) ya indicaba hace una década, el preocupante elevado porcentaje de este tipo de casos que, tal y como muestra el presente trabajo, sigue siendo relevante, especialmente en el caso de los varones. A nivel preventivo, deberían analizarse los factores que influyen en este hecho y en la tendencia encontrada respecto a un mayor número de varones víctimas de este tipo de abuso sexual.

La diferenciación entre abuso sexual con inicio anterior y posterior a los 13 años, ya utilizada por De Paúl y colaboradores (1995), añade información significativa a la descripción de los abusos sexuales. Según los resultados obtenidos, parece ser que el abuso sexual más frecuente es aquél cometido sobre una víctima menor de 13 años por un agresor con una diferencia de edad mínima de cinco años, independientemente del sexo de la víctima.

Respecto al agresor, los resultados también se corresponden con las publicaciones al respecto (Finkelhor, 1994). Antes de los 13 años, la mayoría de agresores suelen pertenecer al entorno cercano de la víctima, siendo principalmente amigos o conocidos en el caso de los estudiantes varones y familiares en el caso de las mujeres. En los abusos con inicio posterior a los 13 años, se incrementa el número de agresores desconocidos en estudiantes varones, así como se introduce con frecuencia la tipología de agresor pareja de la víctima, principalmente en estudiantes de sexo femenino.

En general, las características de los abusos sexuales muestran que, si bien no suelen ir acompañados de violencia física y no se producen heridas en la víctima como resultado del abuso, los porcentajes de penetración son notablemente elevados, especialmente en víctimas varones, indicando un tipo de abuso muy severo y no correspondiéndose con los resultados de otros estudios realizados con muestras universitarias. Los datos de este trabajo indican unos porcentajes de penetración oral, anal y/o vaginal muy superiores a los obtenidos por Bendixen y colaboradores (1994), así como por Oaksford y Frude (2001). Bendixen y colaboradores (1994) hallaron un porcentaje de penetración de 3,7\% en las estudiantes mujeres y $0,4 \%$ en los estudiantes varones, mientras que Oaksford y Frude (2001) encontraron que un 1,4\% de las estudiantes mujeres habían sufrido penetración vaginal. De este modo, la población universitaria femenina analizada ha sufrido penetración en una proporción entre dos y cuatro veces superior a los estudios revisados con muestras similares (un 5,03\% de las estudiantes de la muestra), y en una proporción casi seis veces superior para los hombres (un 5,99\% en el caso de los estudiantes varones). Aunque estos datos deberían ser precisados, se puede interpretar que, en la muestra analizada, la gravedad del abuso ha sido muy elevada en los sujetos de la muestra que han informado haber sido víctimas del abuso sexual en su infancia o, por otro lado, que existe una mayor voluntad de revelar abusos sexuales, incluso aquellos de mayor gravedad.

En síntesis, en este trabajo se han actualizado las cifras de prevalencia del abuso sexual infantil obtenidas en estudios españoles hace más de una década, permitiendo su comparación con estudios recientes de 
otros países e indicando un incremento en el porcentaje de víctimas. Los resultados siguen confirmando que, incluso en muestras universitarias, el abuso sexual infantil es un problema mucho más extendido en la sociedad española de lo que previamente podría estimarse.

\section{Referencias}

American Psychiatric Association. (2002). Diagnostic and statistical manual of mental disorders (4th ed., rev). Washington, DC: Author.

Arruabarrena, M. I., \& De Paúl, J. (1999). Maltrato a los niños en la familia. In Evaluación y tratamiento. Madrid: Pirámide.

Bendixen, M., Muus, K., \& Schei, B. (1994). The impact of child sexual abuse. A study of a random sample of Norwegian students. Child Abuse \& Neglect, 18, 837-847.

Briere, J. (1992). Methodological issues in the study of sexual abuse effects. Journal of Consulting and Clinical Psychology, 60, 196-203.

De Paúl, J., Milner, J. S., \& Múgica, P. (1995). Childhood maltreatment, childhood social support, and child abuse potential in a Basque sample. Child Abuse \& Neglect, 19, 907-920.

Dhaliwal, G. K., Gauzas, L., Antonowicz, D. H., \& Ross, R. R. (1996). Adult male survivors of childhood sexual abuse, prevalence, sexual abuse characteristics, and long-term effects. Clinical Psychology Review, 16, 619-639.

Echeburúa, E., \& Guerricaechevarría, C. (2000). Abuso sexual en la infancia, víctimas y agresores. Un enfoque clínico. Barcelona: Ariel.

Finkelhor, D. (1994). The international epidemiology of child sexual abuse. Child Abuse \& Neglect, 18, 409-417.

Finkelhor, D., \& Hotaling, G. T. (1984). Sexual abuse in the National Incidence Study of Child Abuse and Neglect: An appraisal. Child Abuse \& Neglect, 8, 23-33.

Halperin, D. S., Bouvier, P., Jaffe, P. D., Mounoud, R. L., Pawlak, C. H., Laederach, J., Wicky, H. R., \& Astie, F. (1996). Prevalence of child sexual abuse among adolescents in Geneva: Results of a cross sectional survey. British Medical Journal, $312,1326-1329$.

Kempe, C. H. (1978). Sexual abuse, another hidden pediatric problem, the 1977 C. Anderson Aldrich lecture. Pediatrics, 62, 382-389.

Kubany, E. S., \& Haynes, S. N. (2001). Traumatic Life Events Questionnaire. Manual. Second draft. Western Psychological Services.

López, F. (1994). Los abusos sexuales de menores. Lo que recuerdan los adultos. Madrid: Ministerio de Asuntos Sociales.

MacMillan, H. L. (1998). Child abuse: A community problem. Canadian Medical Association Journal, 158, 1301-1302.

Milner, J. S., Robertson, K. R., \& Rogers, D. L. (1990). Childhood history of abuse and adult child abuse potential. Journal of Family Violence, 5, 15-34.

Oaksford, K. L., \& Frude, N. (2001). The prevalence and nature of child sexual abuse: Evidence from a female university sample in the UK. Child Abuse Review, 10, 49-59.

Rind, B., Tromovitch, P., \& Bauserman, R. (1998). A meta-analytic examination of assumed properties of child sexual abuse using college samples. Psychological Bulletin, 124, 22-53.

Romano, E., \& De Luca, R. V. (2001). Male sexual abuse: A review of effects, abuse characteristics, and links with later psychological functioning. Aggression and Violent Behavior, 6, 55-78.

Runyan, D. K. (1998). Prevalence, risk, sensitivity, and specificity: A commentary on the epidemiology of child sexual abuse and the development of a research agenda. Child Abuse \& Neglect, 22, 493-498.

Violato, C., \& Genuis, M. (1993). Problems of research in male child sexual abuse: A review. Journal of Child Sexual Abuse, 2 , $33-54$. 